\title{
Immunogenicity of a new enhanced tetanus-reduced dose diphtheria-acellular pertussis (Tdap) vaccine against Bordetella pertussis in a murine model
}

\author{
Kyu Ri Kang ${ }^{1}$, Dong Ho Huh ${ }^{1}$, Ji Ahn Kim ${ }^{1}$ and Jin Han Kang ${ }^{1,2^{*}}$ (]
}

\begin{abstract}
Background: The necessity of the tetanus-reduced dose diphtheria-acellular pertussis (Tdap) vaccine in adolescence and adults has been emphasized since the resurgence of small-scale pertussis in Korea and worldwide due to the waning effect of the vaccine and variant pathogenic stains in the late 1990s. GreenCross Pharma (GC Pharma), a Korean company, developed the Tdap vaccine GC3111 in 2010. Recently, they enhanced the vaccine, GC3111, produced previously in 2010 to reinforce the antibody response against filamentous hemagglutinin (FHA). In this study, immunogenicity and efficacy of the enhanced Tdap vaccine compared and evaluated with two Tdap vaccines, GC3111 vaccine produced in 2010 previously and commercially available Tdap vaccine in a murine model.
\end{abstract}

Methods: Two tests groups and positive control group of Balb/c mice were primed with two doses of the diphtheriatetanus-acellular pertussis (DTaP) vaccine followed by a single booster Tdap vaccine at 9 week using the commercially available Tdap vaccine or 2 Tdap vaccines from GC Pharma (GC3111, enhanced GC3111). Humoral response was assessed 1 week before and 2 and 4 weeks after Tdap booster vaccination. The enhanced GC3111 generated similar humoral response compare to the commercial vaccine for filamentous hemagglutinin (FHA). The interferon gamma (IFN-ץ) (Th1), interleukin 5 (IL-5) (Th2) and interleukin 17 (IL-17) (Th17) cytokines were assessed 4 weeks after booster vaccination by stimulation with three simulators: heat inactivated Bordetella pertussis (hBp), vaccine antigens, and hBp mixed with antigens ( $\mathrm{hBp}+$ antigen). A bacterial challenge test was performed 4 weeks after booster vaccination.

Results: Regarding cell-mediated immunity, cytokine secretion differed among the three simulators. However, no difference was found between two test groups and positive control group. All the vaccinated groups indicated a Th1 or Th1/Th2 response. On Day 5 post-bacterial challenge, B. pertussis colonies were absent in the lungs in two test groups and positive control group.

Conclusions: Our results confirmed the immunogenicity of GC Pharma's Tdap vaccine; enhanced GC3111 was equivalent to the presently used commercial vaccine in terms of humoral response as well as cell-mediated cytokine expression.

Keywords: Tetanus-reduced dose diphtheria-acellular pertussis vaccine, Immunogenicity, Mouse study

*Correspondence: kjhan@catholic.ac.kr

${ }^{1}$ The Vaccine Bio Research Institute, College of Medicine, The Catholic University of Korea, Seoul, Annex to Seoul Saint Mary Hospital, 222 Banpo-daero, Seocho-gu, Seoul 06591, Korea

Full list of author information is available at the end of the article

\section{Background}

Sporadic outbreaks of pertussis among adolescents and adults have continuously been reported worldwide, including in advanced countries such as Europe, 
Australia, the USA, and Japan, where the rate of vaccination is above $90 \%$, and yet, the disease is spreading steadily [1-3]. The reasons for the increased occurrence of pertussis include the following. The antibodies produced after acellular pertussis vaccination last for approximately 5-6 years, $[4,5]$, and thus, the likelihood of reinfection increases during adolescence and adulthood because of the waning effect of acellular pertussis vaccine. Moreover, the genetic changes in circulating strains of Bordetella pertussis (B. pertussis) such as pertactin (PRN)-deficient variants [6-8] or pertussis toxin (PT) promoter alleles [9] in advanced countries, may have caused the reemergence of pertussis. In addition, the pertussis vaccination rate reported to be low in adolescents and adults. Furthermore, resistance to macrolide antibiotics following outbreaks is a major challenge in some countries [10]. Therefore, to address the epidemiological changes, the tetanus-reduced dose diphtheria-acellular pertussis (Tdap) vaccination should be encouraged to adolescents and adults, and simultaneously, new vaccines that protect against variant strains should be developed.

In Korea, the Korea National Institution of Health established the laboratory diagnostics of pertussis in 1999 , and since then, only 18 incidences observed annually until 2008. However, the numbers increased subsequently, with 66 cases in 2009, 27 in 2010, and 97 cases observed in 2011 [11]. In the first half of 2012, sudden small outbreaks reported around the schools in certain regions. Since then, small sporadic outbreaks have continued to occur with a steady increasing trend. With epidemiological changes in Korea, immunization of adolescents and adults with Tdap vaccine is necessary [12, 13]. Currently, no Tdap vaccine manufacturer exists in Korea, so the country relies on imported vaccines. Therefore, vaccination is limited, as the vaccine is not easily available. To resolve this issue, Green Cross Pharma (GC Pharma, Yongin, Korea) began developing a Tdap vaccine (GC3111) in 2010 and began Phase I and IIa clinical trials in 2017. During the trials, the antibody titre against PT, filamentous hemagglutinin antigen (FHA), and PRN antigens revealed positive seroconversion and seroprotection after vaccination; however, the vaccine induced a lower titre level of the antibody to FHA compared to the commercially available control vaccine Boostrix ${ }^{\mathrm{TM}}$ (GlaxoSmithKlein, Rixensart, Belgium) [14]. Based on this finding, an enhanced GC3111 Tdap vaccine with increased antigen volume was developed by improving FHA inactivation and purification. The present study aimed to investigate whether the enhanced vaccine (enhanced GC3111) had improved immunological outcomes and efficacy by comparing the vaccine produced in 2010 (GC3111) and the existing commercial vaccine using an animal-based model prior to conducting human trials.

\section{Methods \\ Mice}

During the animal research period, the mouse were housed in filter-top cages under semi-specific pathogen free conditions and food and water are available freely. All animal research procedures performed in accordance with the Laboratory Animals Welfare Act, the Guide for the Care and Use of Laboratory Animals and the Guidelines and Policies for Rodent Experiments provided by the IACUC (Institutional Animal Care and Use Committee) in the School of Medicine, The Catholic University of Korea. (Approval number: CUMS-2019-0100-01).

\section{Vaccination}

The study conducted according to previous murine model studies at our laboratory at the Vaccine Bio Research Institute $[15,16]$. Four-week-old BALB/c female mice from Orient-bio Co., Ltd. (Seongnam, Korea) used in the study. All mice were vaccinated with two doses of primary diphtheria-tetanus-acellular pertussis (DTaP) vaccine at 3-week intervals with $0.125 \mathrm{~mL}$ (one-fourth of the human dose) via intramuscular (quadriceps muscle) injection and vaccinated except for the negative control group, which was injected with saline before booster vaccination. DTaP vaccines were provided by GC Pharma and composed of $25 \mathrm{Lf}$ diphtheria toxoid (DT), $10 \mathrm{Lf}$ tetanus toxoid (TT), $25 \mu \mathrm{g}$ PT, $25 \mu \mathrm{g}$ FHA $25 \mu \mathrm{g}$ and $8 \mu \mathrm{g}$ PRN per $0.5 \mathrm{~mL}$. The mice divided into 4 groups $(30$ mice per group) according to booster vaccine types: negative control injected with saline, positive control with licensed Tdap vaccine (Boostrix ${ }^{\mathrm{TM}}$ ) and two study groups; one study group with the Tdap vaccine GC3111 produced in 2010 by GC Pharma and the other study group with the enhanced FHA antigen of GC 3111 vaccine (Table 1). All Tdap vaccine components were equivalent to DT $2 \mathrm{IU}$, TT $20 \mathrm{IU}, \mathrm{PT} 8 \mu \mathrm{g}$, FHA $8 \mu \mathrm{g}$, and PRN $2.5 \mu \mathrm{g}$ per $0.5 \mathrm{~mL}$ and vaccinated with $0.125 \mathrm{~mL}$ via intramuscular injection. The vaccination and assay schedule described in Fig. 1.

\section{Humoral immune response assay}

Blood samples from the retro-bulbar venous plexuses were collected in each group at 1 week before booster vaccination ( $\mathrm{n}=6$ per group) and 2 , 4 week after booster vaccination $(\mathrm{n}=10)$. When sampling the blood, all mice were anesthetized with tiletamine, zolazepam and xylazine via intra peritoneal injection except last sampling. At 4 week after booster vaccination, mice were euthanized by $2 \%$ isoflurane inhalation while sampling and sacrificed via $\mathrm{CO}_{2}$ inhalation. The 
Table 1 Characteristics of study groups

\begin{tabular}{lll}
\hline Group & Primary (1 st, 2nd) vaccine & Booster vaccine \\
\hline Negative control & Saline & Saline \\
Positive control & DTaP from GC pharma & Commercially available Tdap \\
GC3111 & DTaP from GC pharma & GC3111 Tdap produced in 2010 \\
Enhanced GC3111 & DTaP from GC pharma & GC3111 Tdap FHA enhanced
\end{tabular}

$\mathrm{DTaP}=$ diphtheria-tetanus-acellular pertussis, GC Pharma $=$ Green Cross Pharma, Tdap $=$ tetanus-reduced dose diphtheria-acellular pertussis, $\mathrm{FHA}=$ filamentous hemagglutinin

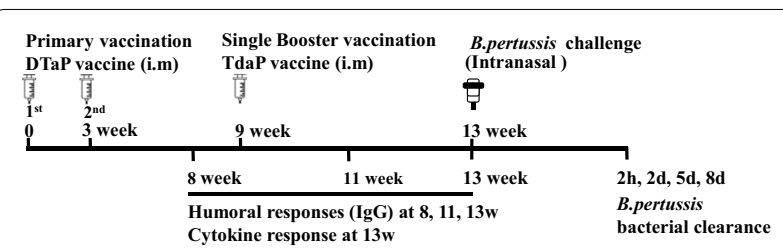

Fig. 1 Schemes for vaccination, challenge and assay of study (DTaP = diphtheria-tetanus-acellular pertussis, Tdap = tetanus-reduced dose diphtheria-acellular pertussis)

humoral immunogenicity against pertussis antigens (anti-PT IgG, anti-FHA IgG and anti-PRN IgG) was evaluated by commercially available ELISA kits (Alpha Diagnostic International Inc., San Antonio, TX, USA). Additionally, anti-diphtheria toxoid (DT) IgG and antitetanus toxoid (TT) IgG titres were measured using commercially available ELISA kits (Alpha Diagnostic International Inc. San Antonio, TX, USA). Results were analysed through optimal density using an Epoch ELISA plate reader (BioTek Instrumetns Inc., Winooski, VT, USA). Antibody titres of each tested antigen compared between groups at each time point.

\section{Cell-mediated immune response assay}

Four weeks after the booster vaccination, mouse spleen cells ( $n=5$ per group) were resuspended in RPMI-1640 (HyClone, GE Healthcare Life Sciences, SouthLogan, Utah, USA) medium containing penicillin, streptomycin, and 10\% FBS. For cell-based experiments, $1 \mu \mathrm{g} / \mathrm{mL}$ pokeweed mitogen (PWM; Sigma-Aldrich, St. Louis, MO, USA) was used as a positive control, and the following three stimulators were tested: $1 \times 10^{6}$ colony forming units (CFUs) /mL of heat inactivated B. pertussis (hBp), PT $(8 \mu \mathrm{g} / \mathrm{mL})$, FHA $(8 \mu \mathrm{g} / \mathrm{mL})$ and PRN $(4 \mu \mathrm{g} / \mathrm{mL})$ vaccine antigens, and the mixture of the two $(\mathrm{hBp}+$ antigens). Splenocytes $\left(5 \times 10^{6}\right.$ cells $\left./ \mathrm{mL}\right)$ of each mice were added to 6-well plates ( $2 \mathrm{~mL} /$ well $)$ and treated with three simulators separately and cultured for 3 days. Subsequently, the cytokine response was assessed by analysing the supernatant using ELISA kits (R\&D Systems, Minneapolis, MN, USA).

\section{Bacterial challenge test}

The protective efficacy against $B$. pertussis infection was assessed with intranasal clearance tests according to previous study [15-17]. The challenge $B$. pertussis strain obtained from a Korean adult pertussis patient was supplied from the Korean Centers for Disease Control \& Prevention (KCDC) (No. 13674) and was inoculated at 4 weeks after booster vaccination. $6 \times 10^{6} \mathrm{CFUs}$ of $B$. pertussis suspended in $50 \mu \mathrm{L}$ of phosphate buffered saline (PBS) and injected intranasally. Four mice in each group at each time point were euthanized by $2 \%$ isoflurane inhalation and their lungs were extracted $2 \mathrm{~h}, 2$ days, 5 days and 8 days after infection. The extracted whole lungs (5 lobes) were grinded with $10 \mathrm{~mL}$ of PBS and diluted to tenfold dilutions. Each diluted homogenate was cultured on Bordet-Gengou agar supplemented with $15 \%$ defibrinated horse blood and incubated for 5 days at $37^{\circ} \mathrm{C}$. CFUs on each media were determined and mean CFUs were compared between groups at each time point.

\section{Statistical analysis}

All results are expressed as the means \pm standard errors of the means (SEM) and compared by two-way ANOVA with Tukey's multiple comparison test. Statistical analysis was performed using GraphPad Prism ${ }^{\text {TM }}$ software v7.02 (GraphPad, San Diego, CA, USA), and statistical significance was defined as a $p$ value $\left(" p<0.05,{ }^{*} p<0.01\right.$, $p<0.001, \quad p<0.0001)$.

\section{Results}

\section{Humoral response}

The humoral immune response was examined 1 week before ( $n=6$ per group) and 2 and 4 weeks $(n=10$ per group) after the booster vaccination. In the antiDT IgG, anti-TT IgG and anti-pertussis IgG humoral responses, the enhanced GC3111 group had equivalent titres compared to the positive control $(p>0.01$, Fig. 2). Titre of two study groups and positive control group had highest titre at 4 weeks after boosting against for PT antigen but for FHA and PRN antigen had highest titre at 2 weeks after boosting (Fig. 2B). The mean titre of anti-PT IgG peaked to 22,270.00 $\mathrm{U} / \mathrm{mL}$ in positive control group and 19,203.90 $\mathrm{U} / \mathrm{mL}$ 

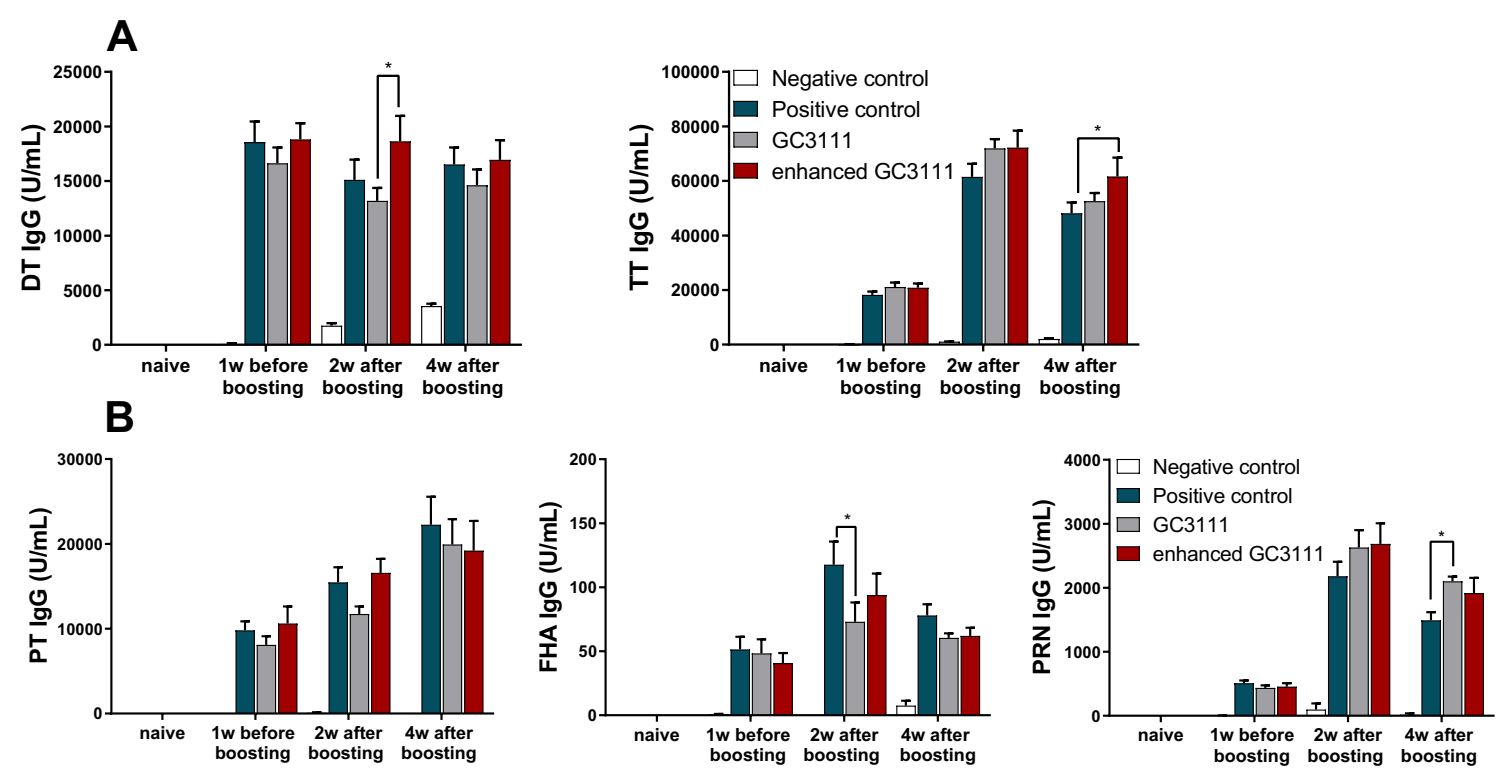

Fig. 2 Humoral immune response. Humoral response was assessed 1 week before and 2 and 4 weeks after the booster vaccination. The results from commercially available ELISA kits are presented as the mean $\pm S E M(U / m L)$ in the graphs. The experiment was performed using five mice per group in the naïve groups and six mice per group 1 week before vaccination. In all other conditions, experiments were performed on 10 mice per group. Statistical differences were tested with two-way ANOVA and Tukey's multiple comparison test. A IgG responses to diphtheria and tetanus were assessed by using commercially available ELISA kits. B Anti-PT, anti-FHA, and anti-PRN IgG titer levels were assessed by using commercially available kits. (SEM = standard errors of the means, PT = pertussis toxin, $\mathrm{FHA}=$ filamentous haemagglutinin, $\mathrm{PRN}=$ pertactin) $\left({ }^{*} p<0.05,{ }^{* *} p<0.01\right.$, $\left.{ }^{* * *} p<0.001,{ }^{* * * *} p<0.0001\right)$

in enhanced GC3111 group at 4 weeks after boosting (Table 2). The mean titre against for FHA antigen had increased 2 weeks after boosting, $117.70 \mathrm{U} / \mathrm{mL}$ in positive control, $93.90 \mathrm{U} / \mathrm{mL}$ in enhanced GC3111 and $73.10 \mathrm{U} / \mathrm{mL}$ in GC3111 group (Table 2). In a similar way to FHA, anti-PRN IgG peaked 2 weeks after boosting, $2,181.00 \mathrm{U} / \mathrm{mL}$ in positive control and 2,686.10 U/ $\mathrm{mL}$ in enhanced GC3111 group (Table 2).

Table 2 Humoral responses against pertussis toxin (PT), filamentous hemagglutinin (FHA), and pertactin (PRN) (mean \pm SEM)

\begin{tabular}{|c|c|c|c|c|}
\hline & Negative control & Positive control & GC3111 & Enhanced GC3111 \\
\hline \multicolumn{5}{|l|}{ Anti-PT IgG (U/mL) } \\
\hline Naïve $(N=5)$ & $0.86 \pm 0.17$ & $0.55 \pm 0.16$ & $0.66 \pm 0.16$ & $0.82 \pm 0.31$ \\
\hline 1 w before booster vaccination $(n=6)$ & 0 & $9,804.25 \pm 1,061.82$ & $8,102.17 \pm 1,002.83$ & $10,608.92 \pm 2,022.17$ \\
\hline 2w after booster vaccination $(\mathrm{N}=10)$ & $92.20 \pm 63.98$ & $15,457.80 \pm 1,781.44$ & $11,734.00 \pm 886.59$ & $16,580.25 \pm 1,663.38$ \\
\hline 4 w after booster vaccination $(N=10)$ & 0 & $22,270.00 \pm 3,286.30$ & $19,936.00 \pm 2,972.66$ & $19,203.90 \pm 3,494.35$ \\
\hline \multicolumn{5}{|l|}{ Anti-FHA IgG (U/mL) } \\
\hline Naïve $(N=5)$ & 0.01 & 0 & 0 & $0.02 \pm 0.01$ \\
\hline 1 w before booster vaccination $(\mathrm{N}=6)$ & $0.88 \pm 0.22$ & $51.41 \pm 9.98$ & $48.39 \pm 11.00$ & $40.74 \pm 7.85$ \\
\hline $2 \mathrm{w}$ after booster vaccination $(\mathrm{N}=10)$ & 0 & $117.70 \pm 17.95$ & $73.10 \pm 15.04$ & $93.90 \pm 16.80$ \\
\hline $4 \mathrm{w}$ after booster vaccination $(\mathrm{N}=10)$ & $7.50 \pm 3.84$ & $78.00 \pm 8.79$ & $60.50 \pm 3.45$ & $62.00 \pm 6.35$ \\
\hline \multicolumn{5}{|l|}{ Anti-PRN IgG (U/mL) } \\
\hline Naïve $(\mathrm{N}=5)$ & 0 & 0 & 0 & 0 \\
\hline 1 w before booster vaccination $(N=6)$ & $2.88 \pm 2.80$ & $510.42 \pm 42.25$ & $436.08 \pm 38.92$ & $456.75 \pm 52.19$ \\
\hline $2 \mathrm{w}$ after booster vaccination $(\mathrm{N}=10)$ & $96.40 \pm 96.40$ & $2,181.00 \pm 225.35$ & $2,629.10 \pm 272.59$ & $2,686.10 \pm 320.84$ \\
\hline $4 \mathrm{w}$ after booster vaccination $(\mathrm{N}=10)$ & $18.90 \pm 18.90$ & $1,491.50 \pm 127.52$ & $2,101.40 \pm 73.77$ & $1,918.10 \pm 237.37$ \\
\hline
\end{tabular}

$\mathrm{SEM}=$ standard errors of the means 


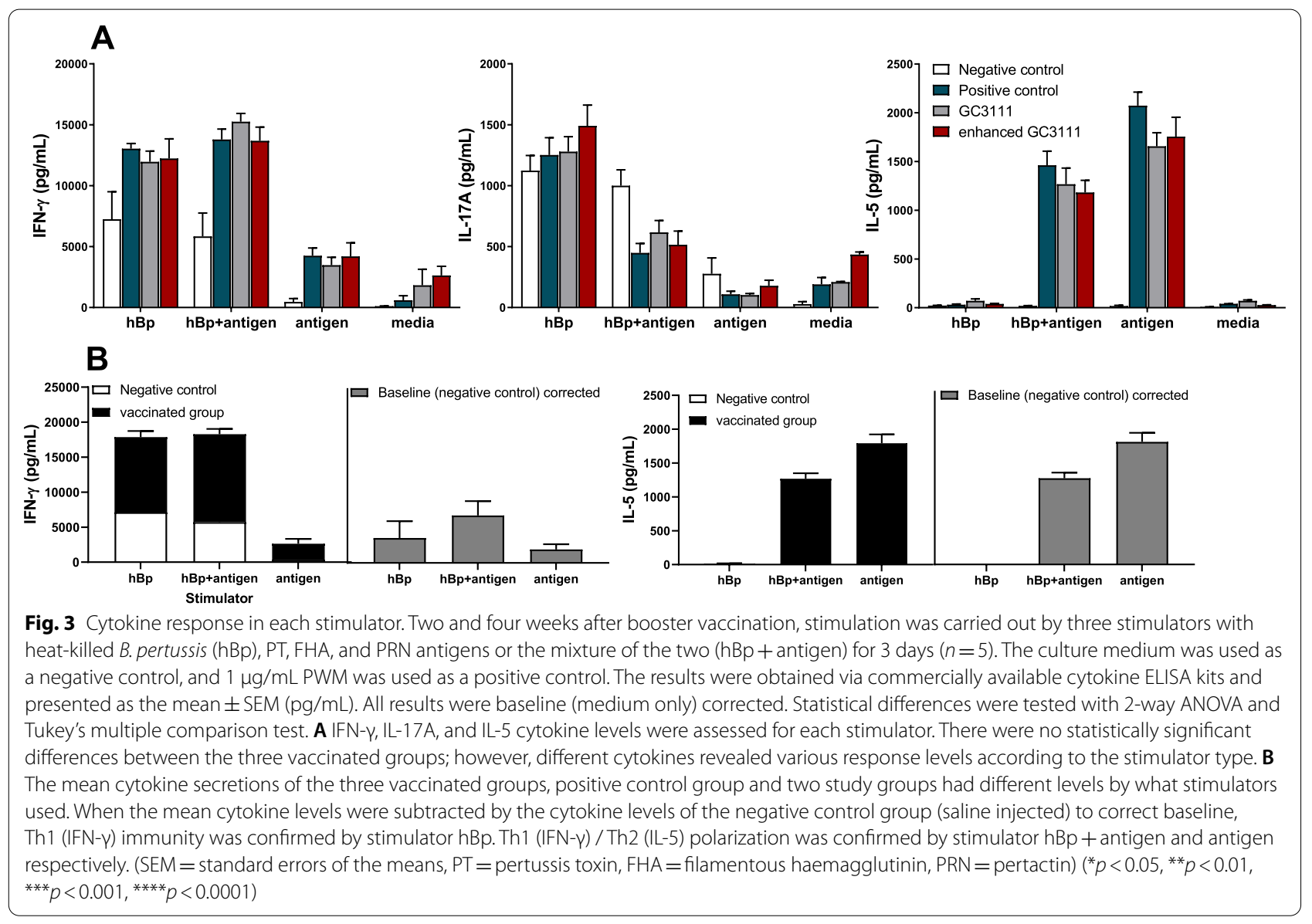

\section{Cell-mediated immune response}

Cell-mediated immunity (CMI) was evaluated by stimulating mouse splenocytes ( $\mathrm{n}=5$ per group) to $\mathrm{hBp}$, vaccine antigens or $\mathrm{hBp}+$ antigens, whereas the culture medium was used as a negative control, and $1 \mu \mathrm{g} /$ $\mathrm{mL}$ PWM was used as a positive control. The results showed that the secretion of IFN- $\gamma$, IL-17A and IL-5 did not differ significantly among the vaccinated groups except for the media negative control group and PWM control group ( $p>0.05$, Fig. 3A). However, cytokine secretion was significantly different according to the stimulator used; the mean IFN- $\gamma$ expression levels of the three vaccinated groups, stimulated by $\mathrm{hBp}+$ antigen was three times higher than that of the antigenstimulated group. Furthermore, IL-5 was significantly upregulated in the groups stimulated by the vaccine antigens, while limited responses were observed when stimulated by hBp (Fig. 3A,B). On the other hand, IL17A had no responses because the negative control group overexpressed by non-specific inflammation response by all three stimulators. In this study, the CMI of the vaccinated groups confirmed Th1/Th2 immunity in both $\mathrm{hBp}+$ antigen and antigen stimulator (Fig. 3B).
The immunity polarization was calculated with mean cytokine levels of three vaccinated groups (positive control and two study groups) that were baseline corrected with media stimulated results and negative control (saline injected) group results. When hBp stimulator used, only IFN- $\gamma$ showed response as $3582.48 \mathrm{pg} /$ $\mathrm{mL}$ in the three vaccinated groups (Fig. 3B). The mean cytokine response of the three vaccinated groups was $6826.51 \mathrm{pg} / \mathrm{mL}$ and $1247.40 \mathrm{pg} / \mathrm{mL}$ for IFN- $\gamma$ and IL-5, respectively when stimulated with $\mathrm{hBp}+$ antigen (Fig. 3B). IFN- $\gamma$ showed $1943.97 \mathrm{pg} / \mathrm{mL}$ and IL-5 showed $1773.25 \mathrm{pg} / \mathrm{mL}$ when stimulated with antigen (Fig. 3B).

\section{Bacterial clearance in lung}

The vaccine efficacy was evaluated against the clinical pertussis strain. Four mice was sacrificed at each time points per group. The results from the test using the clinically isolated strain showed that B. pertussis was removed quickly in the lungs and was almost eliminated after 5 days (Fig. 4). The results were the same in the two study groups and the positive control group. Compare to $2 \mathrm{~h}$ after intranasal challenge, the CFUs of B. pertussis 


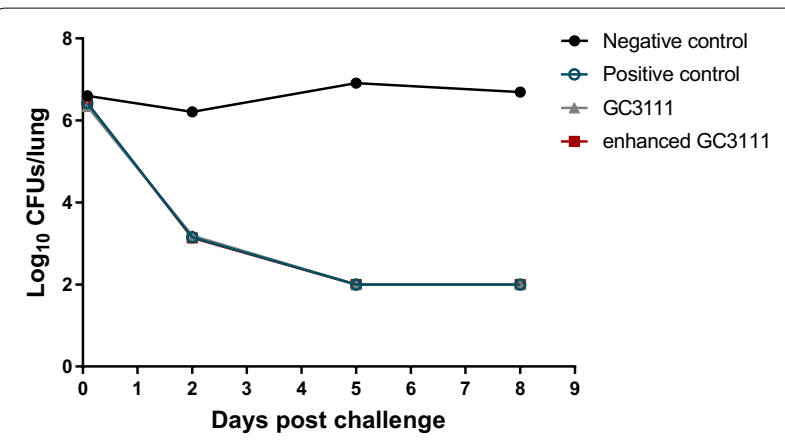

Fig. 4 Bacterial clearance in lung. Lungs were extracted from the mice subjected to the challenge test, and the pertussis bacterial colonies were enumerated at $2 \mathrm{~h}$ and 2, 5, and 8 days after the challenge. Four mice were sacrificed at each time points. By Day 5 post-challenge, the bacterial colonies were hardly found in any of the groups, with the exception of the negative control group, which was injected with saline solution

decreased at day 2 in the study groups and positive control group (Fig. 4). This result showed protective efficacy against $B$. pertussis in both the positive group and the two study groups while the negative control group retained bacterial CFUs during the test period and showed even more CFUs than $2 \mathrm{~h}$ after challenge.

\section{Discussion}

Since 2000, a serological study in Korea has confirmed incidents of pertussis infection with higher certainty than reported earlier, and small-scale pertussis outbreaks have occurred once every 3 years since 2009, leading to the requirement for Tdap vaccination. In 2010, GC Pharma, a national company, started developing Tdap and DTaP vaccines; and our laboratory, the Vaccine Bio Research Institute, conducted animal-based studies $[15,16]$ and performed clinical trials [14] using the Tdap booster vaccine. In animal studies, GC Pharma's new Tdap vaccine, GC3111, was compared with Boostrix ${ }^{\text {TM }}$, a commercially available product in Korea. The humoral immunity was assessed after a single dose of DTaP vaccine followed by Tdap booster vaccine [15], and CMI was assessed after two doses of DTaP vaccine followed by Tdap booster vaccine [16]. After two animal studies and clinical trials, GC Pharma complemented GC3111 to improve the anti-FHA antibody response. The present study aimed to show the immunogenic response and efficacy of complemented GC3111 (enhanced GC3111) compared to Boostrix $^{\mathrm{TM}}$ and the former GC3111 vaccine and to verify anti-FHA response reinforcement.

The protective effects of the humoral response to the aP vaccine were actively investigated soon after aP vaccine development, and the importance of the humoral response to PT, PRN, FHA, and fimbriae antigens was evaluated in different systems, including animal models [18-20]. Humoral immunity to these antigens of B. pertussis is known to protect the individual from pertussis infection by neutralizing the pathogenic antigens or by activating the complement system that activates CMI [20-22]. Among the immunogens present in the vaccine, PT is known as the most important immunogen and induces the generation of protective antibodies that provide direct protection from pertussis infection [23, 24]. Moreover, the humoral immunity generated by aPvaccinated pregnant women can prevent infants from pertussis infection since the antibodies produced by the mother can deliver to the foetus [25-28]. Thus, evaluation of humoral response after pertussis vaccination is immensely significant. In this study, compared to the positive control, the GC3111 group showed lower antiFHA antibody level 2 weeks after booster vaccination, which was in line with the observations of a previous study [14]; however, the antibody response was comparable between the enhanced GC3111 group and the positive control group with respect to all antigens and all time points (Fig. 2B). Hence, our results verified that the humoral immune response was improved with the enhanced GC3111 Tdap vaccine. With respect to the effect of two doses of DTaP vaccination, anti-PT, antiFHA, and anti-PRN antibody titres were elevated even before the booster vaccination, and these levels were further enhanced after the booster vaccination and retained at a high level until 4 weeks after vaccination (Fig. 2B). Considering the humoral responses to tetanus and diphtheria, all groups except the negative control group revealed a titre of over $0.1 \mathrm{U} / \mathrm{mL}$ (the protective level) from 1 week before booster vaccination, and this is predicted by two doses of DTaP vaccination (Fig. 2A).

The fact that CMI plays crucial roles in preventing pertussis infection was first shown in a mouse model in 1993 [29] followed by clinical experiments [30]. The importance of both Th1 [31, 32] and Th17 [33-35] type CMI responses was confirmed in animal and clinical studies. IFN- $\gamma$ and IL-17 are the main cytokines that provide crucial protection. Recently, the resurgence of pertussis outbreaks [1-3] and the protective effects of the aP vaccine and whole cell pertussis $(\mathrm{wP})$ vaccine [36] were compared frequently. This was based on the observations of some researchers who postulated that the $\mathrm{wP}$ vaccine induces Th1/Th17 responses similar to natural infection $[33,37,38]$, whereas the aP vaccine mainly induces the Th2 response, resulting in a weaker protective effect than that of the $\mathrm{wP}$ vaccine. Previous studies showed that the aP vaccine generated Th1/Th2 [39] or Th2/Th17 [38, 40] responses. This inconsistency in the findings may be attributed to the difference in animal models used in the studies [41] as well as the study design, such as vaccine 
schedule and stimulation condition. In general, the Th2 dominant response is the common CMI in aP vaccinebased studies. In this study, hBp was included as one of the simulators to indirectly examine the effects of natural exposure, while vaccine antigens were used as simulators to evaluate the response to the aP vaccine. After exposure to the stimulators, IFN- $\gamma$, IL-17, and IL-5 showed significant differences between stimulators that were used but no differences between the positive control group and study groups (Fig. 3A). In the negative control group, non-specific inflammation response was observed in the IFN- $\gamma$ and IL-17 cytokines. Particularly, IL-17 cytokine showed no responses because the negative control group and vaccinated groups had similar results regardless of the stimulators (Fig. 3A). Notably, in the hBp stimulator group, we observed only IFN $-\gamma$ responses (Fig. 3B). In the $\mathrm{hBp}+$ antigen or antigen stimulator group, the levels of IFN- $\gamma$ and IL- 5 were significantly higher compared to the saline injected negative control group, indicating that Th1 (IFN- $\gamma$ )/Th2 (IL-5) adaptive immunity (Fig. 3B). This result is consistent with previous studies showing that the aP vaccine primarily induces the Th2 response but induces a dominant Th1 response when exposed to natural pertussis [42-45]. However, in this study, there are some limitations due to the in vitro system, and natural pertussis exposure could be substituted by $\mathrm{hBp}$ indirectly.

In addition, the results of the bacterial challenge test using the clinical pertussis strain for real and reliable assessment showed that all vaccinated groups cleared the pathogen from Day 2 post-challenge, and by Day 5 , the pathogen was hardly found in the lungs, thereby confirming the similar efficacy of the booster vaccines in the three vaccinated groups (Fig. 4).

\section{Conclusions}

GC Pharma's enhanced GC311 Tdap vaccine addresses the limitations of the previous GC311 Tdap vaccine, wherein a lower anti-FHA antibody response is observed compared to that of the commercially available product. Our study outcomes confirmed that after booster vaccination, the humoral as well as the CMI responses were comparable to those of the commercially available product with equivalent efficacy against the clinical strain. Our findings present strong evidence that similar findings may be obtained in the phase II clinical trial that is currently being carried out.

\footnotetext{
Abbreviations

aP: Acellular Bordetella pertussis; CFU: Colony forming unit; CMI: Cell mediated immunity; DT: Diphtheria toxoid; DTaP: Diphtheria-tetanus-acellular pertussis; FHA: Filamentous hemagglutinin; PRN: Pertactin; PT: Pertussis toxin; PWM: Pokeweed mitogen; SEM: Standard errors of the means; Tdap: Tetanus-reduced dose diphtheria-acellular pertussis; TT: Tetanus toxoid; WP: Whole cell pertussis.
}

\section{Supplementary Information}

The online version contains supplementary material available at https://doi. org/10.1186/s12865-021-00457-1.

Additional file 1. Table S1. Raw data of cell mediated cytokine immune response by cytokine ELISA kit.

Additional file 2. Table S2. B. pertussis clearance was evaluated. The colony forming cells from lungs were counted two times.

\section{Acknowledgements}

Not applicable.

\section{Authors' contributions}

JHK and DHH designed the study. JAK, KRK and DHH conducted the experiments and analysed the data. KRK and JHK wrote the manuscript. All authors read and approved the final manuscript.

\section{Funding}

This study was supported by Green Cross Pharma (Yongin, Korea, Grant No.52018-D0083-0001, 5-2019-D0083-00002). The funding bodies played no role in the design of the study and collection, analysis, and interpretation of data and in writing the manuscript.

\section{Availability of data and materials}

The datasets used and/or analyzed during the current study are available from the corresponding author on reasonable request.

\section{Declarations}

Consent to publication

Not applicable.

\section{Ethics approval}

All animal research procedures were performed in accordance with the Laboratory Animals Welfare Act, the Guide for the Care and Use of Laboratory Animals and the Guidelines and Policies for Rodent Experiments provided by the IACUC (Institutional Animal Care and Use Committee) in the School of Medicine, The Catholic University of Korea. (Approval number: CUMS-2019-0100-01).

\section{Competing interests}

There is no conflicts of interest in this study.

\section{Author details}

${ }^{1}$ The Vaccine Bio Research Institute, College of Medicine, The Catholic University of Korea, Seoul, Annex to Seoul Saint Mary Hospital, 222 Banpo-daero, Seocho-gu, Seoul 06591, Korea. ${ }^{2}$ Department of Pediatrics, Seoul St. Mary's Hospital, College of Medicine, The Catholic University of Korea, 222 Banpo-daero, Seocho-gu, Seoul 06591, Korea.

Received: 1 December 2020 Accepted: 28 September 2021 Published online: 12 October 2021

References

1. Winter K, et al. California pertussis epidemic, 2010. J Pediatr. 2012;161(6):1091-6.

2. Cherry JD. Epidemic pertussis in 2012-the resurgence of a vaccinepreventable disease. N Engl J Med. 2012;367(9):785-7.

3. Chiappini $E$, et al. Pertussis re-emergence in the post-vaccination era. BMC Infect Dis. 2013;13:151

4. Wendelboe A, et al. Duration of immunity against pertussis after natural infection or vaccination. Pediatr Infect Dis J. 2005:24:S58-61.

5. Klein NP, et al. Waning protection after fifth dose of acellular pertussis vaccine in children. N Engl J Med. 2012;367(11):1012-9. 
6. Hegerle N, et al. Evolution of French Bordetella pertussis and Bordetella parapertussis isolates: increase of Bordetellae not expressing pertactin. Clin Microbiol Infect. 2012;18(9):E340-6.

7. Zeddeman A, et al. Investigations into the emergence of pertactin-deficient Bordetella pertussis isolates in six European countries, 1996 to 2012. Euro Surveill. 2014;19:33.

8. Lam C, et al. Rapid increase in pertactin-deficient Bordetella pertussis isolates, Australia. Emerg Infect Dis. 2014;20(4):626-33.

9. Mooi FR, et al. Bordetella pertussis strains with increased toxin production associated with pertussis resurgence. Emerg Infect Dis. 2009;15(8):1206-13.

10. Li L, et al. High prevalence of macrolide-resistant Bordetella pertussis and ptxP1 genotype, Mainland China, 2014-2016. Emerg Infect Dis. 2019;25(12):2205-14

11. Lee SY, et al. Pertussis prevalence in Korean adolescents and adults with persistent cough. J Korean Med Sci. 2015;30(7):988-90.

12. Kwon HJ, et al. Infant pertussis and household transmission in Korea. J Korean Med Sci. 2012;27(12):1547-51.

13. Lee $S Y$, et al. Pertussis seroprevalence in korean adolescents and adults using anti-pertussis toxin immunoglobulin G. J Korean Med Sci. 2014;29(5):652-6.

14. Park C, et al. Development and implementation of standardized method for detecting immunogenicity of acellular pertussis vaccines in Korea. Clin Exp Vaccine Res. 2019;8(1):35-42.

15. Kwon HJ, et al. Assessment of safety and efficacy against Bordetella pertussis of a new tetanus-reduced dose diphtheria-acellular pertussis vaccine in a murine model. BMC Infect Dis. 2017;17(1):247.

16. Han SB, et al. Preliminary study on the immunogenicity of a newly developed GCC Tdap vaccine and its protection efficacy against Bordetella pertussis in a murine intranasal challenge model. Clin Exp Vaccine Res. 2015;4(1):75-82.

17. Huh DH, et al. Immunogenicity and protective efficacy of a newly developed tri-component diphtheria, tetanus, and acellular pertussis vaccine in a murine model. J Microbiol Immunol Infect. 2018;51(6):732-9.

18. Boursaux-Eude C, et al. Intranasal murine model of Bordetella pertussis infection: II. Sequence variation and protection induced by a tricomponent acellular vaccine. Vaccine. 1999;17(20-21):2651-60.

19. Bruss JB, Siber GR. Protective effects of pertussis immunoglobulin (P-IGIV) in the aerosol challenge model. Clin Diagn Lab Immunol. 1999;6(4):464-70.

20. Cherry JD, et al. A search for serologic correlates of immunity to Bordetella pertussis cough illnesses. Vaccine. 1998;16(20):1901-6.

21. Vidarsson G, Dekkers $G$, Rispens T. IgG subclasses and allotypes: from structure to effector functions. Front Immunol. 2014;5:520.

22. Storsaeter J, et al. Levels of anti-pertussis antibodies related to protection after household exposure to Bordetella pertussis. Vaccine. 1998;16(20):1907-16.

23. Giammanco A, et al. Analogous IgG subclass response to pertussis toxin in vaccinated children, healthy or affected by whooping cough. Vaccine. 2003;21(17-18):1924-31.

24. Taranger J, et al. Correlation between pertussis toxin IgG antibodies in postvaccination sera and subsequent protection against pertussis. J Infect Dis. 2000;181(3):1010-3.

25. Bechini $A$, et al. Acellular pertussis vaccine use in risk groups (adolescents, pregnant women, newborns and health care workers): a review of evidences and recommendations. Vaccine. 2012;30(35):5179-90.

26. Winter $\mathrm{K}$, et al. Effectiveness of prenatal versus postpartum tetanus, diphtheria, and acellular pertussis vaccination in preventing infant pertussis. Clin Infect Dis. 2017;64(1):3-8.
27. Campbell $\mathrm{H}$, et al. Review of vaccination in pregnancy to prevent pertussis in early infancy. J Med Microbiol. 2018;67(10):1426-56.

28. Mazzilli S, Tavoschi L, Lopalco PL. Tdap vaccination during pregnancy to protect newborns from pertussis infection. Ann Ig. 2018;30(4):346-63.

29. Mills KH, et al. Cell-mediated immunity to Bordetella pertussis: role of Th1 cells in bacterial clearance in a murine respiratory infection model. Infect Immun. 1993;61(2):399-410.

30. Ausiello CM, et al. Cell-mediated immunity and antibody responses to Bordetella pertussis antigens in children with a history of pertussis infection and in recipients of an acellular pertussis vaccine. J Infect Dis. 2000;181(6):1989-95.

31. Mahon BP, et al. Atypical disease after Bordetella pertussis respiratory infection of mice with targeted disruptions of interferon-gamma receptor or immunoglobulin mu chain genes. J Exp Med. 1997;186(11):1843-51.

32. Barbic J, et al. Role of gamma interferon in natural clearance of Bordetella pertussis infection. Infect Immun. 1997;65(12):4904-8.

33. Warfel JM, Zimmerman LI, Merkel TJ. Acellular pertussis vaccines protect against disease but fail to prevent infection and transmission in a nonhuman primate model. Proc Natl Acad Sci USA. 2014;111(2):787-92.

34. Khader SA, et al. IL-23 and IL-17 in the establishment of protective pulmonary CD4+ T cell responses after vaccination and during Mycobacterium tuberculosis challenge. Nat Immunol. 2007;8(4):369-77.

35. Fedele G, Bianco M, Ausiello CM. The virulence factors of Bordetella pertussis: talented modulators of host immune response. Arch Immunol Ther Exp (Warsz). 2013;61(6):445-57.

36. Brummelman J, et al. Roads to the development of improved pertussis vaccines paved by immunology. Pathog Dis. 2015;73(8):ftv067.

37. Vermeulen $F$, et al. Cellular immune responses of preterm infants after vaccination with whole-cell or acellular pertussis vaccines. Clin Vaccine Immunol. 2010;17(2):258-62.

38. Ross PJ, et al. Relative contribution of Th1 and Th17 cells in adaptive immunity to Bordetella pertussis: towards the rational design of an improved acellular pertussis vaccine. PLoS Pathog. 2013;9(4):e1003264.

39. Reynolds $E$, et al. Laboratory investigation of immune responses to acellular pertussis vaccines when used for boosting adolescents after primary immunisation with whole cell pertussis vaccines: a comparison with data from clinical study. Vaccine. 2006;24(16):3248-57

40. Brummelman J, et al. Modulation of the CD4(+) T cell response after acellular pertussis vaccination in the presence of TLR4 ligation. Vaccine. 2015:33(12):1483-91.

41. Kapil P, Merkel TJ. Pertussis vaccines and protective immunity. Curr Opin Immunol. 2019:59:72-8.

42. Ausiello CM, et al. Cell-mediated immune responses in four-year-old children after primary immunization with acellular pertussis vaccines. Infect Immun. 1999;67(8):4064-71.

43. He Q, et al. Cytokine mRNA expression and proliferative responses induced by pertussis toxin, filamentous hemagglutinin, and pertactin of Bordetella pertussis in the peripheral blood mononuclear cells of infected and immunized schoolchildren and adults. Infect Immun. 1998;66(8):3796-801.

44. Ryan M, et al. Distinct T-cell subtypes induced with whole cell and acellular pertussis vaccines in children. Immunology. 1998;93(1):1-10.

45. Edwards KM, Berbers GA. Immune responses to pertussis vaccines and disease. J Infect Dis. 2014;209(Suppl 1):S10-5.

\section{Publisher's Note}

Springer Nature remains neutral with regard to jurisdictional claims in published maps and institutional affiliations. 